\title{
PVA Microporous Hydrospheres
}

National Cancer Institute

\section{Source}

National Cancer Institute. PVA Microporous Hydrospheres. NCI Thesaurus. Code C71147.

An embolic material composed of microspheres of polyvinyl alcohol (PVA) polymers.

These water-soluble, compressible microspheres may be used to encapsulate various therapeutic agents; drug-loaded microspheres can then be used as a drug delivery vehicle during embolization of tumor vasculature. 